\title{
A Novel Reliability Oriented Bi-Objective Unit Commitment Problem
}

\author{
Ali Azizivahed \\ Department of Electrical and Electronics Engineering, Shiraz \\ University of Technology, Shiraz, Iran \\ a.azizi@sutech.ac.ir
}

\begin{abstract}
This paper presents a new solution to unit commitment for single-objective and multi-objective frameworks. In the first step, the total expected energy not supplied (TEENS) is proposed as a separate reliability objective function and at the next step, the multi-objective Pareto front strategy is implemented to simultaneously optimize the cost and reliability objective functions. Additionally, an integer based codification of initial solutions is added to reduce the dimension of ON/OFF status variables and also to eliminate the negative influence of penalty factor. The modified invasive weed optimization (MIWO) algorithm is also developed to optimally solve the proposed problem. The obtained solutions are compared with results in the literature which confirms the applicability and superiority of the proposed algorithm for a 10-unit system and 24-hour scheduling horizon.
\end{abstract}

Index Term - Unit commitment, bi-objective optimization, the total expected energy not supplied (TEENS), invasive weed optimization (MIWO) algorithm.

\section{INTRODUCTION}

Unit commitment (UC) is one of the most important optimization problems in the power system operation and management [1]. This problem should be solved for achieving an appropriate scheduling of generation power units. Improvement of the commitment scheduling of units results in the reduction of generation cost. Although this improvement might seem small, it can provide significant profits in remarkable scales of power generation. Therefore, the main aim of the UC problem at each time interval is to recognize a combination of generation units to serve the system demand at a minimum cost subject to a number of equality and inequality constraints [2].

On the other hand, the most economical combination of generation units is not necessarily satisfactory from a reliability point of view. In other words, the optimal generation schedule for operation cost minimization might jeopardize the system reliability level. Toward this end, researches have been recently directed toward obtaining the optimal generation schedules that simultaneously satisfy different objectives. Therefore, a hybrid multi-objective approach is proposed in this study to solve UC as a multi-objective optimization problem considering generation cost and TEENS as two

\author{
Sahand Ghavidel, Mojtaba Jabari Ghadi, Li Li and Jiangfeng \\ Zhang \\ Faculty of Engineering and Information Technology, University \\ of Technology Sydney, PO Box 123, Broadway, NSW 2007, \\ Australia
}

objective functions.

The UC problem consists of some operational constraints such as minimum up and down times and ramp rate constraints which increases the complexity degree of the problem [3-5].

UC inherently is a complex mixed integer quadratic programming problem which needs to be solved by a robust and efficient optimization algorithm. Up to now, different mathematical techniques including priority list (PL), dynamic programming (DP), mixed integer programming (MIP), Lagrangian relaxation (LR) and branch and bound [6, 7] have been implemented to solve the UC problem. It is notable that, these deterministic techniques are computationally inefficient. The UC as one of the most complicated optimization problems in power system operation area also gets more complicated by extending as a multi-objective optimization problem and considering different practical constraints. Therefore, it is crucial to recognize an appropriate and powerful optimization algorithm for the UC problem. Proposing new algorithms for solving UC problem has become a broad potential of research. Evolutionary algorithms (EAs) are well-known optimization methods to deal with non-linear, mixed integer, and complex problems.

The shuffle frog leaping algorithms (SFLA) for solving the UC problem is developed and compared with the results of different EAs in [6]. A quasi-oppositional teaching learningbased optimization (QOTLBO) algorithm is introduced in [8] for solving the UC problem considering spinning reserve and ramp rate of generating unit.

The invasive weed optimization (IWO) is efficacious EA which has been successfully applied to different optimization problems. This algorithm is inspired from the colonization of invasive weeds in nature which is firstly proposed in [9]. According to the nature of the stochastic evolutionary processes, IWO is computationally expensive. Furthermore, it has some drawbacks such as trapping in local optimal points and premature convergence in some cases. Toward this end, a mutation strategy is proposed in this paper to ameliorate the deficiencies of the original IWO algorithm. Considering in this paper two different objective functions (cost and reliability), which are in conflict, has been proposed as a multi-objective 
UC problem; it should obtain a set of optimal solutions instead of one. In this regard, a repository is utilized to save all nondominated solutions (Pareto-optimal solutions). Also, a fuzzy decision-making strategy is used for sorting all Pareto-optimal solutions based on their importance.

In summary, the main contributions of this paper are listed as follows:

- To apply a method for generating units' commitment statuses without using penalty factors to satisfy the ON/OFF status of units and eliminate its negative influences on the number of feasible statuses and convergence speed. The main benefit of this approach is that it can provide feasible and reliable commitment statuses in a short computational time by implementing a smaller set of integer number to generate commitment statuses instead of using large binary matrix. The proposed method can reduce the size of decision variables to have better numerical results.

- To apply TEENS index as a separate objective function in order to obtain a reliable solution for the UC problem. The forced outage rate (FOR) and the reparation time are considered to evaluate the system reliability for each commitment status and power generations. The reliability index (TEENS) and operational cost are optimized simultaneously by implementing multi-objective Pareto front strategy.

- To implement a modified IWO algorithm to obtain optimal solutions. A mutation strategy is employed to improve the performance of the original IWO algorithm and avoid trapping in the local optimal solution.

\section{Proposed Unit COMMITMENT FormUlation AND METHODOLOGY}

Formulation and mathematical modeling of the total generation cost and TEENS objective functions, as well as the system and unit constrains of the proposed study are as follows:

\section{A. Operating costs}

The main objective function of the UC problem is energy generation cost. The generation cost is defined as follows [10]:

$$
\begin{aligned}
& \text { Cost }=\sum_{t=1}^{24}\left(F_{t}+S U_{t}+S D_{t}\right) \\
& F_{t}=\sum_{n=1}^{N_{G}}\left(a_{n} \times P_{n, t}^{2}+b_{n} \times P_{n, t}+c_{n}\right) \times S_{n}^{t} \\
& S U_{t}+S D_{t}=\sum_{n=1}^{N_{G}}\left(s u c_{n, t}+s d c_{n, t}\right)
\end{aligned}
$$

where $F_{t}$ and $S U / S D_{t}$ are generation cost and start-up/shutdown cost at $t^{\text {th }}$ interval; $a_{n}, b_{n}$ and $c_{n}$ are the fuel cost coefficients of $n^{\text {th }}$ unit; $P_{n, t}$ and $S_{n}^{t}$ are the active power and state of $n^{t h}$ unit at $t^{t h}$ hour (0 for OFF and 1 for ON units), respectively; $N_{G}$ is number of generating units; $s u c_{n, t}$ and $s d c_{n, t}$ represent the formulation for start-up and shut-down costs of $n^{\text {th }}$ generator at $t^{\text {th }}$ interval, respectively which can be defined as follows:

$$
\begin{aligned}
& s u c_{n, t}=\left\{\begin{array}{ccc}
C_{S U_{n}} & \text { if } & S_{n}^{t}-S_{n}^{t-1}=1 \\
0 & \text { if } & S_{n}^{t}-S_{n}^{t-1}<1
\end{array}\right. \\
& s d c_{n, t}=\left\{\begin{array}{ccc}
C_{S D_{n}} & \text { if } & S_{n}^{t}-S_{n}^{t-1}=-1 \\
0 & \text { if } & S_{n}^{t}-S_{n}^{t-1}>-1
\end{array}\right.
\end{aligned}
$$

where $C_{S U_{n}}$ and $C_{S D_{n}}$ are the startup and shut down costs of unit $n$, respectively.

\section{B. Reliability assessment}

As mentioned, reliability analysis is one of the main contributions of this paper. In this regard, the TEENS is evaluated as a reliability objective function. The main reason for this choice is that the other reliability indices such as loss of load probability (LOLP) index indicate only the probability of system failure [11]. Therefore, TEENS is considered to cover the wide aspects of power systems' reliability. TEENS can be defined as follows [11]:

$$
\begin{aligned}
& \operatorname{FOR}_{j}=\frac{\lambda_{j}}{\lambda_{j}+\mu_{j}}=\frac{M T T R_{j}}{M T T F_{j}+M T T R_{j}} \\
& \operatorname{MTTR}_{j}=\frac{1}{\mu_{j}} \\
& \operatorname{MTTF}_{j}=\frac{1}{\lambda_{j}}
\end{aligned}
$$

where $F O R_{j}$ is the force outage rate of $j^{\text {th }}$ generator, $\lambda_{j}$ and $\mu_{j}$ are the failure rate and repair rate of $j^{\text {th }}$ generator, respectively; $M T T F_{\mathrm{j}}$ and $M T T R_{j}$ are the mean time of failure and mean time of repair of $j^{\text {th }}$ generator, respectively.

The probability of each status of generating units is defined according to the FOR values of generators as follows:

$$
\operatorname{Prob}_{i}=\prod_{k \in V} F O R_{k} \times \prod_{h \in W}\left(1-F O R_{h}\right)
$$

where $\boldsymbol{V}(\boldsymbol{W})$ is the set of available (unavailable) generating units at $i^{\text {th }}$ load point.

The expected energy not supplied (EENS) of each time interval and each load point is calculated as follows:

$$
\text { EENS }_{i, t}=\left\{\begin{array}{cl}
\left(P l_{t, i}-P_{G, i}\right) \cdot \text { Prob }_{i} & \text { if } P l_{t, i}>P_{G, i} \\
0 & \text { Otherwise }
\end{array}\right.
$$

where $P l_{t, i}$ is the amount of load demand at $t^{t h}$ time interval and $i^{\text {th }}$ load point; $P_{G, i}$ is a summation of power output of available generating units at $i^{\text {th }}$ load point, and $E E N S_{i, t}$ is the amount of energy not supplied at $i^{\text {th }}$ load point and $t^{\text {th }}$ time interval.

The expected energy not supplied for all load points and 24hour time duration is calculated as follows:

$$
\text { TEENS }=\sum_{t=1}^{24} \sum_{i=1}^{L P} E E N S_{i, t} \times T_{i}
$$

where $L P$ is the total number of load points; TEENS is the total amount of expected energy not supplied which is considered as the reliability objective function; $T_{i}$ is the time duration of $i^{\text {th }}$ load point which can be obtained from the 


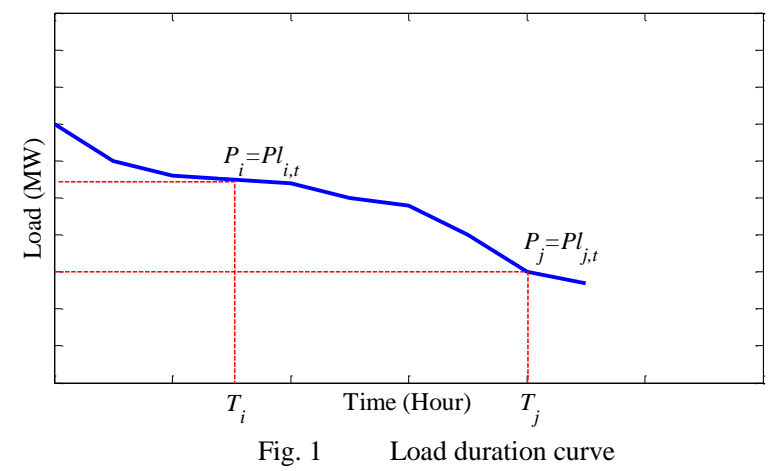

load duration curve. Fig. 1 shows an illustration of load duration curve and two load points with their related time duration. According to this figure, the time duration for two different load points is shown. This time duration (i.e. $T_{i}$ ) shows the number of hours that energy consumption is greater than a particular load points (i.e. $P_{i}=P l_{i, t}$ ).

\section{Limitations and constraints}

All equality and inequality constraints related to the proposed UC problem are represented as follows:

- Generation and load balance

$\forall t \sum_{n=1}^{N_{G}}\left(P_{n, t} \times S_{n}^{t}\right)=P_{l, t}$

- $\quad$ Power generation capacity and ramp rate limits

$P_{n}^{\min } \leq P_{n, t} \leq P_{n}^{\max } \quad$ if $S_{n}^{t}=1, \quad \forall n, t$

$-\operatorname{Ramp}_{\text {Down }, n} \leq P_{n, t}-P_{n, t-1} \leq \operatorname{Ramp}_{U p, n}$

$$
\text { if } S_{n}^{t}-S_{n}^{t-1}=0, \quad \forall n, t
$$

- Reserve constraint

$\forall t \sum_{n=1}^{N_{G}}\left(P_{n}^{\max } \times S_{n}^{t}\right)=P_{l, t}+R_{t}$

- Minimum up and down times

$\forall n, t \begin{cases}\sum_{i=t-T_{O N_{n}}}^{t-1} S_{n}^{i}=T_{O N_{n}} & \text { if }\left(S_{n}^{t}-S_{n}^{t-1}=-1\right) \\ \sum_{i=t-T_{O F F_{n}}}^{t-1} S_{n}^{i}=0 & \text { if }\left(S_{n}^{t}-S_{n}^{t-1}=+1\right)\end{cases}$

where $P_{n}^{\max }\left(P_{n}^{\min }\right)$ and $\mathrm{R}_{\mathrm{t}}$ are the maximum (minimum) power limits of $n^{\text {th }}$ generator and the forecasted spinning reserve at $t^{t h}$ interval, respectively; $T_{O N_{n}}$ and $T_{O F F}$ are the minimum up time and minimum down time of $n^{\text {th }}$ generator, respectively.

\section{Proposed commitment status variables}

In order to have a set of feasible variables which satisfy the minimum up-time and down-time constraints, penalty factors are applied. However, it is worth mentioning that the penalty factor has a negative influence on convergence speed and obtaining the optimal solution. In this regard, a new integer variable coding approach (penalty factor free) is presented to reduce the commitment decision variables' dimension.

The proposed integer variable is defined as follows:

$$
\begin{aligned}
& G(i, d)=\left\{\begin{array}{cc}
-\operatorname{round}\left((1+\operatorname{rand}) \times T_{O F F_{i}}\right) & \text { if } r=0 \\
\operatorname{round}\left((1+\operatorname{rand}) \times T_{O N_{i}}\right) & \text { if } r=1
\end{array}\right. \\
& r=\operatorname{rand}(0 \text { or } 1)
\end{aligned}
$$

It should be mentioned that the minimum up-time and down-time constraints are satisfied automatically because all generated integer variables are bigger than the lower bounds. ALGORITHM-I shows how to generate initial population, which provides a better understanding of the proposed approach. Also, Fig. 2 displays an illustration of the proposed integer variables.

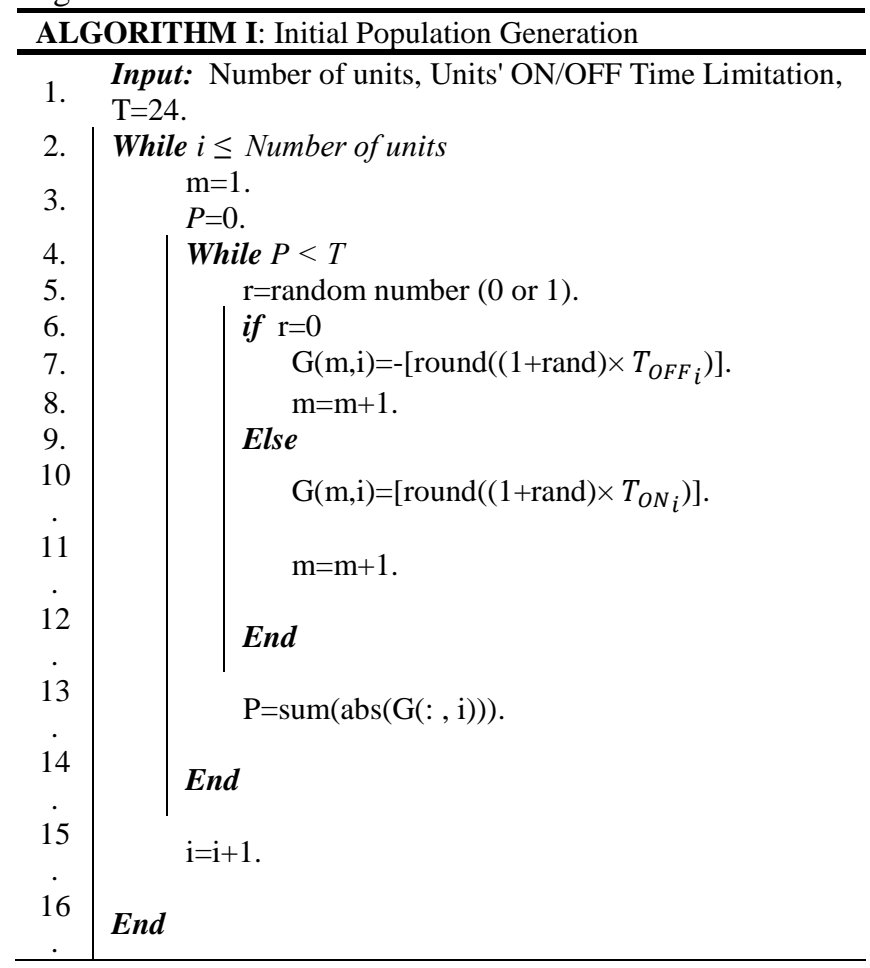

\section{OPTIMIZATION TOOL}

In this paper, the IWO algorithm is implemented as an optimization technique to find the optimal solution. This algorithm is a novel population-based numerical stochastic technique and inspired from the colonization of invasive weeds in nature [9]. The weeds produce the seeds according to their finesses. The weed which has more fitness value produces the maximum number of seeds, while the weed with minimum fitness value produces the minimum number of seeds.

$$
S N(i)=S N_{\text {max }}-\operatorname{abs}\left(\text { floor }\left(\frac{F_{\text {gbest }}-F_{i}}{F_{\text {gbest }} F_{\text {worth }}} \times S N_{\max }\right)\right)
$$

where $S N(i)$ and $S N_{\text {max }}$ are the number of seeds produced by $i^{\text {th }}$ weed and the maximum number of seeds, respectively. $F_{\text {gbest }}\left(F_{\text {worth }}\right)$ is the best (worth) fitness.

The seeds produced around their relative weed based on a normal distribution with mean (i.e. equals 0) and standard deviation which is decreased by an increase in the iteration. 


$$
\sigma_{\text {iter }}=\left(\sigma_{\text {initial }}-\sigma_{\text {final }}\right) \times\left(\frac{\left(\text { iter }_{\max }-\text { iter }\right)}{\text { iter }_{\max }}\right)^{n}
$$

where $\sigma_{i t e r}$ and $n$ (i.e. equals to 5) are the standard deviation of particular iteration and nonlinear modulation index, respectively. $\sigma_{\text {initial }}$ (i.e. equals to 2 ) and $\sigma_{\text {final }}$ (equals to $0.0001)$ are initial and final standard deviation, respectively. iter and iter $_{\max }$ are the number of iteration and maximum predetermined iteration number, respectively.

\begin{tabular}{|c|c|}
\hline \multicolumn{2}{|r|}{ ALGORITHM II: Modified IWO application on UC problem } \\
\hline 1 & Input: Algorithm parameters (i.e. Number of Population \\
\hline 2 & $\begin{array}{l}\text { The weeds (initial population) are initialized depending upon } \\
\text { the } \boldsymbol{A L G O R I T H M - I} \text {. } \\
\text { Iter }=1 \text {. }\end{array}$ \\
\hline 3 & While iter $<$ Maximum iteration \\
\hline 4 & $\begin{array}{l}\text { The seed number is calculated according to Eq. (18). } \\
i=0 \text {. }\end{array}$ \\
\hline 5 & While $i<$ Number of Population \\
\hline 6 & Calculate the standard division according to Eq. (19). \\
\hline 7 & $\begin{array}{l}\text { The seeds are produced around their relative weed } \\
\text { based on normal distribution. } \\
i=i+1 \text {. }\end{array}$ \\
\hline 8 & End \\
\hline 9 & $\begin{array}{l}\text { Calculate the fitness function for each produced seeds } \\
\text { Apply mutation strategy. }\end{array}$ \\
\hline 11 & $\begin{array}{l}\text { Sort all weeds and produced seeds according to the } \\
\text { fitness function value. }\end{array}$ \\
\hline 12 & $\begin{array}{l}\text { Keep } N_{\text {weed }} \text { of populations (which have the best fitness) } \\
\text { as a new weeds. } \\
\text { iter }=\text { iter }+1 \text {. }\end{array}$ \\
\hline 13 & End \\
\hline
\end{tabular}

The mathematical formulation of mutation employed in this framework is shown as follows:

$$
X_{\text {Mut }}=X^{\text {Gbest }}+\varphi \times\left(X_{\text {rand } 1}-X_{\text {rand } 2}\right)
$$

where $X^{\text {Gbest }}$ is the best solution in each iteration. $X_{\text {rand } 1} \neq$ $X_{\text {rand2 }}$ are randomly selected mutant seeds. $\varphi$ is mutation constant which is normally equal to 2 [12].

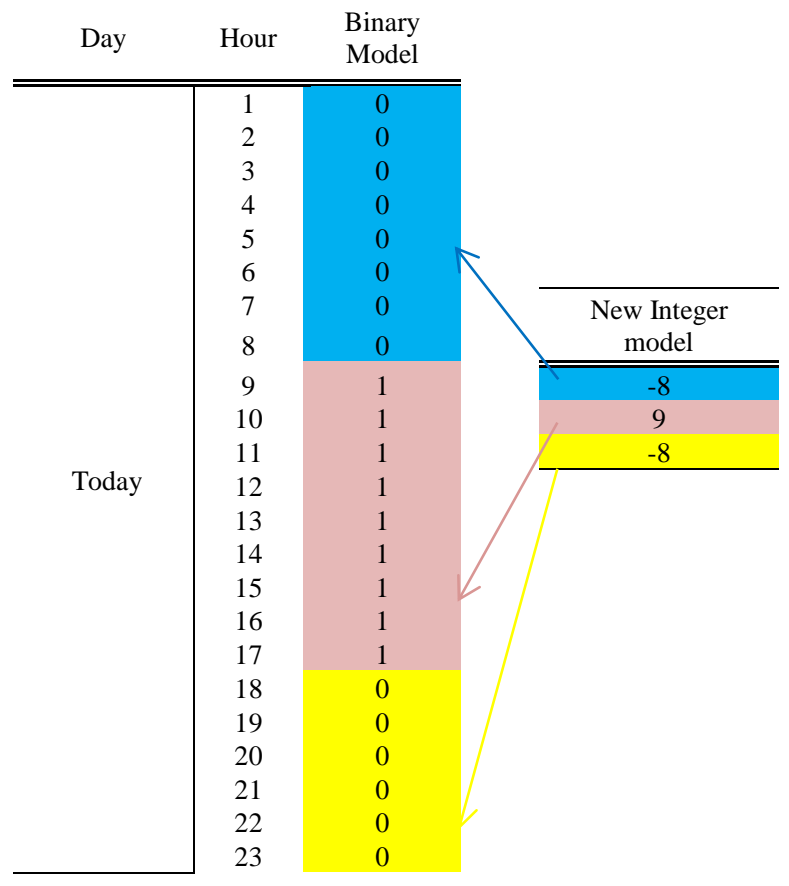

\begin{tabular}{c|cc} 
& 24 & 0 \\
\hline Tomorrow & 1 & 0 \\
\hline
\end{tabular}

Fig. 2 A simple example of proposed integer variable converted to its binary equivalent

TABLE I. COMPARISON OF BEST COST FOR 10UNIT TEST SYSTEM

\begin{tabular}{ll}
\hline \hline Algorithms & Cost $(\$)$ \\
\hline PSO [1] & 565804.00 \\
BGSA [13] & 563937.00 \\
TLBO [8] & 564402.90 \\
QOTLBO [8] & 564394.00 \\
GHS-JGT [14] & 563937.68 \\
BSA [15] & 563937.70 \\
Proposed Approach & $\mathbf{5 6 3 6 3 7 . 1 9}$ \\
\hline \hline
\end{tabular}

The proposed method application on UC problem is presented in ALGORITHM-II.

\section{Simulation Results}

The system under study is a 10-unit generating system. Details of this case study are available in [16]. In order to confirm the ability of the proposed commitment status variables and MIWO algorithm in solving UC and more specifically multi-objective UC problems, three different scenarios are considered for the proposed test system in this study which is listed below:

Case 1: Cost is minimized individually;

Case 2: TEENS is minimized individually;

Case 3: Cost and TEENS are minimized simultaneously; Case I (Cost objective function)

In the first part of numerical simulation, in order to show the capability of proposed method, a comparison between the obtained result of MIWO algorithm and those reported in the literature including PSO [1], BGSA [13], TLBO [8], QOTLBO [8], GHS-JGT [14] and BSA [15] algorithms, for Case I are provided in TABLE I. According to this table, it is obvious that the proposed algorithm can converge to a better solution comparing to the other optimization algorithms. Furthermore, the contribution of each generator related to the optimal cost objective function is depicted in Fig. 3.

\section{E. Case II (TEENS objective function)}

The two objective functions, cost and TEENS, are in conflict. In order to have an optimal solution with minimum cost, the best option is to meet the load with the units with the least generation cost; while, in order to have a more reliable operation scheme, it is better to meet load with more reliable units. Fig. 4 depicts the contribution of units in scheduling with optimal TEENS. The value of TEENS in the cost based UC scheme is 24511152.02 (MWh/year), while this value is 6782722.07 (MWh/year) in TEENS objective based case which shows a significant improvement. Similarly, there is a big gap between cost values in this case and previous case which shows $8.64 \%$ increase in the amount of cost. 


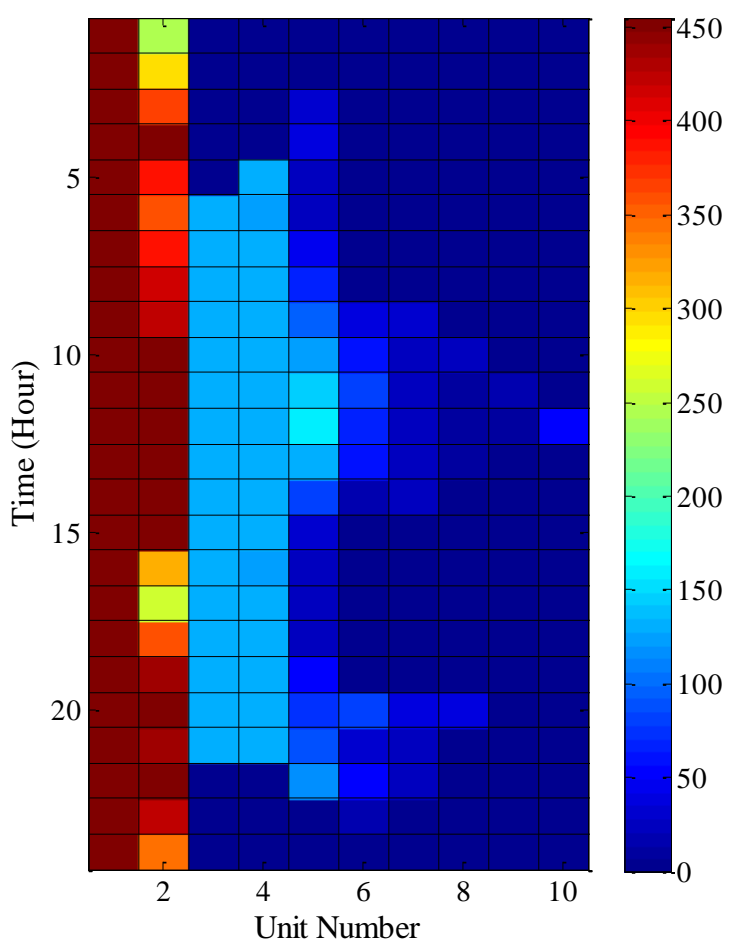

Fig. 3 The power scheduling in optimal cost

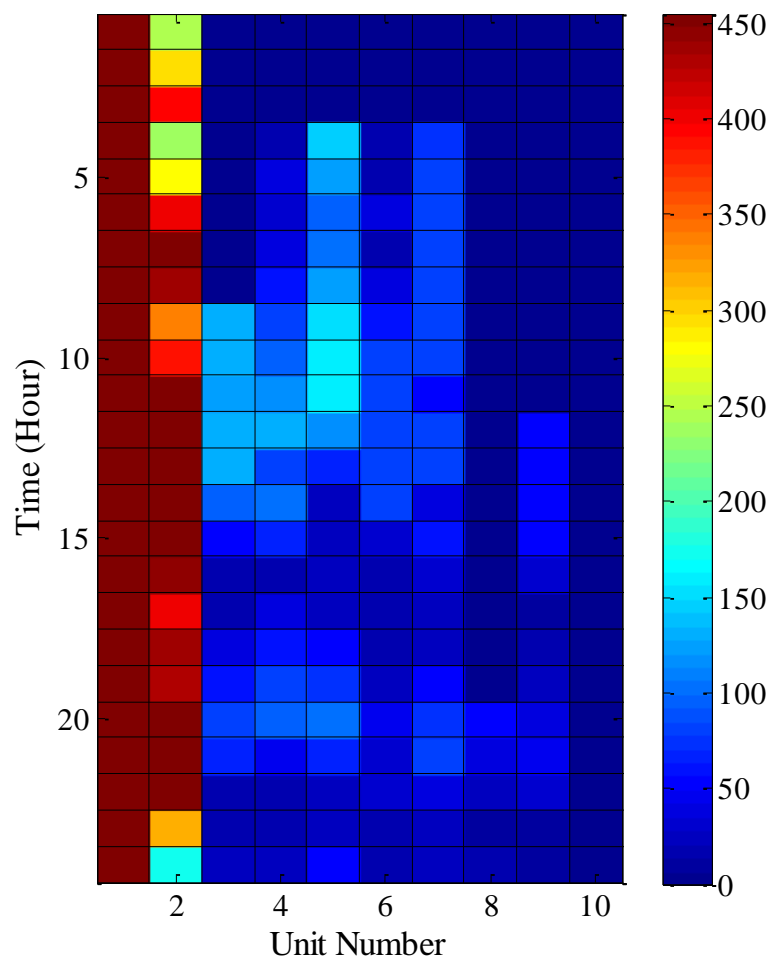

Fig. 4 The power scheduling in optimal TEENS

In this condition, the generators' commitment schedule during the 24-h time horizon is different compared with that in the optimal cost scenario. In this scenario, some expensive and more reliable generating units are committed only to increase the system reliability. However, in optimal cost based scenario, due to the decreasing generation cost, the expensive units are not committed.

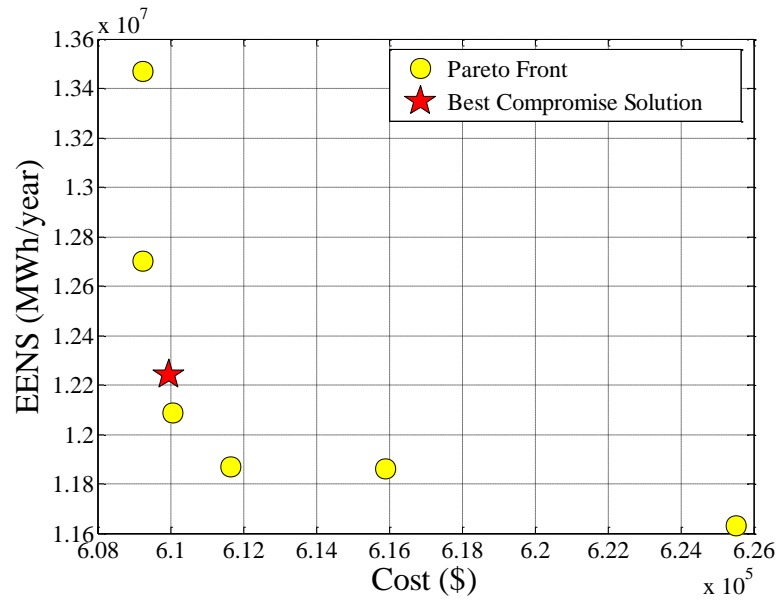

Fig. 5 Two-dimensional Pareto-solutions for cost \& TEENS objectives

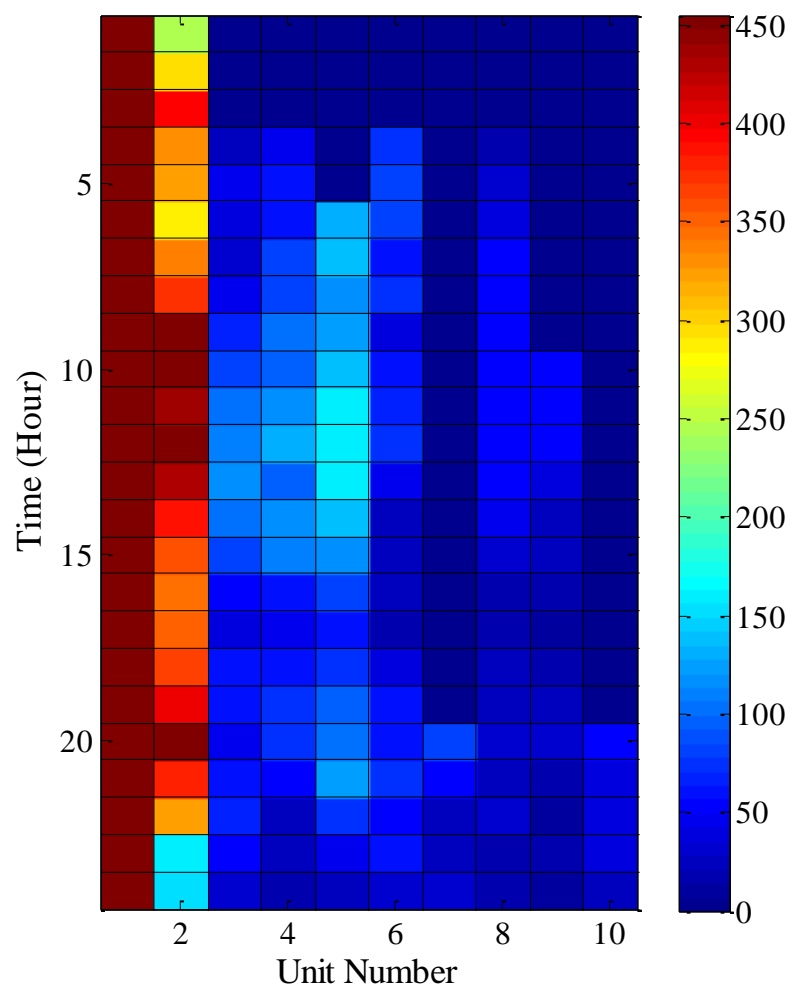

Fig. 6 The power scheduling in best-compromised solution

\section{F. Case III (Multi-objective UC)}

In this case, the bi-objective optimization problem is solved using the MIWO algorithm. A convex Pareto-optimal front including best proposed compromised solution (i.e. red star) is shown in Fig. 5. This set of non-dominated solutions satisfies the operation requirements for power system operators. It is also noted that the convex character of the Pareto-optimal front becomes well visible to show the conflicted relationship between two proposed objective functions. As can be seen from Fig. 5, a lower TEENS generally corresponds to a higher cost. For instance, to have minimum cost $\$ 6.093 \times 10^{5}$, the TEENS value is $1.346 \times 10^{7}(\mathrm{MWh} /$ year). Similarly, in order to minimize the TEENS $1.16 \times 10^{7}$ (MWh/year), the cost value is $\$ 6.254 \times 10^{5}$. 


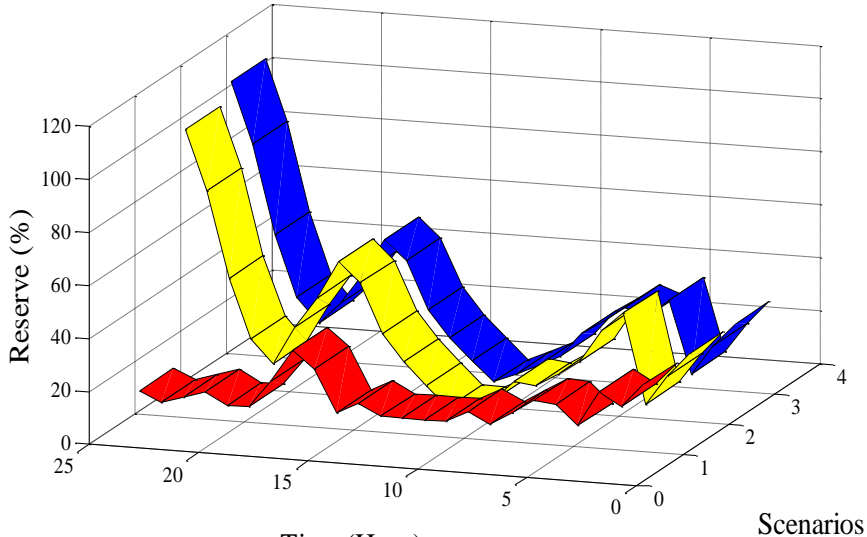

Time (Hour)

Fig. 7 The percentage of reserve for three scenarios; scenario-1 (optimal cost), scenario-2 (optimal TEENS) and scenario-3 (best compromised solution)

According to the obtained set of non-dominated solutions, a best compromised solution can be selected by the system operator according to the practical requirements as well as technical constraints.

The cost and TEENS values in the best compromise solution found by the MIWO algorithm are $\$ 609958.0825$ and 12238877.42 (MWh/year), respectively. The commitment statuses and output power scheduling of this case are more reliable than that of output power scheduling in optimal cost case.

The optimal active power scheduling and percentage of reserves are shown in Fig. 6 and Fig. 7, respectively. Fig. 6 shows the dispatched power of generating units which are committed to the best compromised solution. It can be seen that number of commitment in this situation is more than the previous solutions (minimum cost and minimum TEENS cases). From this figure, it is obvious that a set of units which are committed in minimum cost solution or minimum TEENS solution is committed to a best compromised solution to have a reasonable cost as well as reliability level. Fig. 7 shows that the reserve constraint is satisfied in all three solutions. The reserve percentage in scenarios corresponding to minimum TEENS and the best compromised solution is more than the reserve percentage in case of minimum cost.

\section{CONCLUSION}

In this paper, a bi-objective UC framework to reach optimal solutions of generation cost versus reliability values, based on units' forced outage rate considering some technical constraints, has been developed. TEENS is modeled as the reliability objective function besides the generation cost. An integer codification of commitment variables with high capability to satisfy all the constraints especially the ON/OFF time has been employed aiming at generating the initial population and also simultaneous eliminating negative impacts of penalty factor. The capability of proposed approach with MIWO algorithm has been confirmed by comparing its results with the results of previous researches. Furthermore, the Pareto-front strategy is employed to find a set of non- dominated optimal solutions. The results provide an approach to show how the power system operator is able to select a best compromised solution from the Pareto-optimal front in order to have a good tradeoff between two conflicted objective functions.

\section{REFERENCES}

B. Saravanan, E. Vasudevan, and D. Kothari, "Unit commitment problem solution using invasive weed optimization algorithm," International Journal of Electrical Power \& Energy Systems, vol. 55, pp. 21-28, 2014.

B. Saravanan, C. Kumar, and D. Kothari, "A solution to unit commitment problem using fire works algorithm," International Journal of Electrical Power \& Energy Systems, vol. 77, pp. 221-227, 2016.

R. Azizipanah-Abarghooee, T. Niknam, F. Bavafa, and M. Zare, "Short-term scheduling of thermal power systems using hybrid gradient based modified teaching-learning optimizer with black hole algorithm," Electric Power Systems Research, vol. 108, pp. 16-34, 2014.

A. J. Wood and B. F. Wollenberg, Power generation, operation, and control. John Wiley \& Sons, 2012.

M. Carrión and J. M. Arroyo, "A computationally efficient mixedinteger linear formulation for the thermal unit commitment problem," IEEE Transactions on power systems, vol. 21, no. 3, pp. 1371-1378, 2006.

J. Ebrahimi, S. H. Hosseinian, and G. B. Gharehpetian, "Unit commitment problem solution using shuffled frog leaping algorithm," IEEE Transactions on Power Systems, vol. 26, no. 2, pp. 573-581, 2011.

A. Castillo, C. Laird, C. A. Silva-Monroy, J.-P. Watson, and R. P. O'Neill, "The unit commitment problem with AC optimal power flow constraints," IEEE Transactions on Power Systems, vol. 31, no. 6, pp. 4853-4866, 2016.

P. K. Roy and R. Sarkar, "Solution of unit commitment problem using quasi-oppositional teaching learning based algorithm," International Journal of Electrical Power \& Energy Systems, vol. 60, pp. 96-106, 2014.

S. Karimkashi and A. A. Kishk, "Invasive weed optimization and its features in electromagnetics," IEEE transactions on antennas and propagation, vol. 58, no. 4, pp. 1269-1278, 2010.

A. Shukla and S. N. Singh, "Multi-objective unit commitment using search space-based crazy particle swarm optimisation and normal boundary intersection technique," IET Generation, Transmission \& Distribution, vol. 10, no. 5, pp. 1222-1231, 2016.

R. Billinton, H. Chen, and R. Ghajar, "Time-series models for reliability evaluation of power systems including wind energy," Microelectronics Reliability, vol. 36, no. 9, pp. 1253-1261, 1996.

K. P. Wong and Z. Y. Dong, "Differential evolution, an alternative approach to evolutionary algorithm," in Intelligent Systems Application to Power Systems, 2005. Proceedings of the 13th International Conference on, 2005, pp. 73-83: IEEE.

X. Yuan, B. Ji, S. Zhang, H. Tian, and Y. Hou, "A new approach for unit commitment problem via binary gravitational search algorithm," Applied Soft Computing, vol. 22, pp. 249-260, 2014.

N. Kumar, B. K. Panigrahi, and B. Singh, "A solution to the ramp rate and prohibited operating zone constrained unit commitment by GHS-JGT evolutionary algorithm," International Journal of Electrical Power \& Energy Systems, vol. 81, pp. 193-203, 2016.

F. Bavafa, T. Niknam, R. Azizipanah-Abarghooee, and V. Terzija, "A New Biobjective Probabilistic Risk-Based Wind-Thermal Unit Commitment Using Heuristic Techniques," IEEE Transactions on Industrial Informatics, vol. 13, no. 1, pp. 115-124, 2017.

S. A. Kazarlis, A. Bakirtzis, and V. Petridis, "A genetic algorithm solution to the unit commitment problem," IEEE transactions on power systems, vol. 11, no. 1, pp. 83-92, 1996. 